\title{
The Smooth Curvature Flexure Model: An Accurate, Low-dimensional Approach for Robot Analysis
}

\author{
Lael U. Odhner and Aaron M. Dollar \\ Yale University \\ Email: \{lael.odhner, aaron.dollar\}@yale.edu
}

\begin{abstract}
This paper presents a new and comprehensive method for modeling robots having highly flexible members such as flexure joints. An accurate model of large deformation bending is important for precisely describing the configuration of the flexible member. Additionally, the accuracy of the Jacobian and Hessian of the forward kinematics are critically important at large angles for predicting the deformation and the stiffness of the joint under load. The model introduced here is based on the assumption that the curvature of a beam in bending is smooth, and thus can be approximated by low-order polynomials. This produces a parameterized description of flexure motion that can be used as a joint model when expressed in Denavit-Hartenberg form, as a transformation from one rigid link to the next in a serial manipulator. We will show that with only three parameters, this model faithfully reproduces the elastic deformation of a flexure hinge predicted by the continuum model, even for large angles, without requiring numerical integration or many finite elements. It can also be used to compute the compressive buckling load of the flexure as predicted by the continuum model.
\end{abstract}

\section{INTRODUCTION}

Highly flexible members have been frequently considered in the context of robotic hardware. A number of studies into the behavior of flexible link robots have been conducted, often for the purposes of controlling for undesirable dynamic effects related to working with long, thin links (e.g. [1-3]). A smaller number of efforts have dealt with the beneficial aspects of highly flexible links, such as providing a large number of degrees of freedom for manipulation tasks [4] or low stiffness for grasping and assembly purposes [5]. A related application is the use of highly flexible members as joints between rigid links, typically referred to as flexures. The contrast between these two applications is shown in Fig. 1. These features are commonly used to allow motion in monolithic structures, and have been used as joints in a number of different robotic mechanisms, particularly in compliant hands [6-8]. The benefits of flexure-based joints include having no sliding parts (and therefore no friction or stick-slip effects), no backlash, and are able to compliantly deform in response to unplanned collisions, making them ideal for robots that must operate in unstructured environments [9].

Another major benefit of flexures is the simplicity and potential lower cost compared to standard revolute joints, which require bearings for smooth, accurate motion. As robots become more common as commercial products, flexures are

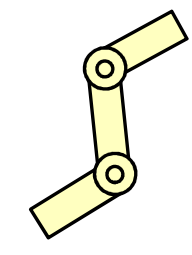

Rigid Links Pin Joints

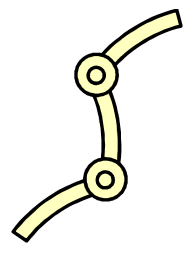

Flexible Links Pin Joints

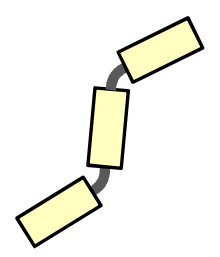

Rigid Links Flexure Joints
Figure 1. A comparison between traditional rigid robot manipulators (left), flexible link robots (center), and flexure-joint robots, in which flexible links act as hinges (right).

likely to be used with increasing frequency due to their compatibility with inexpensive polymeric fabrication processes such as multi-shot injection molding [10] and shape deposition manufacturing [11].

One drawback to flexure-based robot mechanisms is the complex mechanical behavior that they exhibit compared to pin joints. A pin joint has one degree of freedom, whereas the elastic deformation of a beam in bending has infinitely many degrees of freedom. Moreover, a flexure hinge in a robot often bends to angles up to 90 degrees or more, so classical smalldeflection beam bending models are inapplicable. As a result, there is no canonical parametric model for planar flexure hinges suitable for robot analysis. This paper presents a model that can fill this role. In order to apply the rich set of tools available for serial manipulator design, control, and analysis, one must have a model of elastic behavior that is accurate and computationally simple. The design specification for a good flexure model can be broken into three functional requirements:

1. It should be possible to compute both the shape of the robot and the elastic energy associated with deformation as a function of a small set of generalized coordinates, as one might describe a jointed mechanism using the internal joint angles.

2. It should be possible to compute the force in generalized coordinates resulting from a force on the robot at any point using the Jacobian of that point's coordinates, as well as the local equilibrium position resulting from such a force.

3. It should be possible to compute the stiffness in generalized coordinates resulting from a force on the robot at a point by the Hessian of that point's coordinates, as well as any buckling modes the robot has (configurations/loads having zero stiffness in some direction). 
Many models of flexible robot components meet some, but not all of these requirements. One common approach is to model flexure elements as having constant curvature [12]. Flexures have also been approximated as a single pin joint halfway between the ends of the flexure [13]. Both of these models capture the relative rotation between rigid bodies on the manipulator, making them useful for inverse kinematic computation and form closure grasp analysis. However, because these models have fewer degrees of freedom than a real flexure, they are too rigid and under-predict the deformation of a loaded flexure. Another family of models, called pseudo-rigid body models, consists of one or several joints placed to approximate the flexure's center of rotation, connected in parallel with nonlinear springs fit by regression to the exact force-deflection profile [14]. These models can be used to find flexure deflection under load, but they are unsuitable for manipulator analysis because the linkage geometries used to approximate the beam bending change based on the direction of applied force, and thus are not purely kinematic descriptions of flexure behavior. Another approach is based on assuming some set of superimposed deformational modes [15]. This technique has been applied to flexible links (e.g. [16]) and continuum manipulators [17]. Modal models for flexures have been proposed based on analytically calculated small-deformation solutions, as well as finite element solutions $[18,19]$. However, modal models are specific to the behavior they are designed to model, and none of the currently available models in the robotics literature accurately capture largedeformation flexure behavior.

The flexure model discussed in this paper is a modal model which approximates the curvature of a flexure using a polynomial basis. The polynomial coefficients define the relative position and orientation of two bodies connected by the flexure, as well as the elastic energy stored in the flexure itself. This model meets all three of the functional requirements introduced above, while avoiding the need to use numerical integration or to break the beam into many finite elements. It predicts not only the deflection of a flexure under load, but also second-order kinematic effects such as buckling and the change in flexure stiffness resulting from compressive or tensile loads. These second-order effects are particularly useful in the study of grasping and manipulation, where grasp stability may depend on the elastic stability of the manipulator itself [20]. Previous study of this model has dealt with stiffness prediction [21]. This paper is intended as a more general discussion of this model's applicability to robotic systems.

The remainder of this paper is divided into three sections. Section II is an overview of the flexure model, describing how the parameters define the shape and the energy function of a flexure hinge. Section III examines the shape of the flexure when an arbitrary load and moment is applied at one end. The results are compared to exact large deformation beam solutions. Additional results are shown comparing the smooth curvature model to finite element models of a sample mechanism. Section IV demonstrates the second-order kinematic accuracy of the model by comparing classical continuum buckling models to the discrete buckling predicted by the proposed parameterized model.
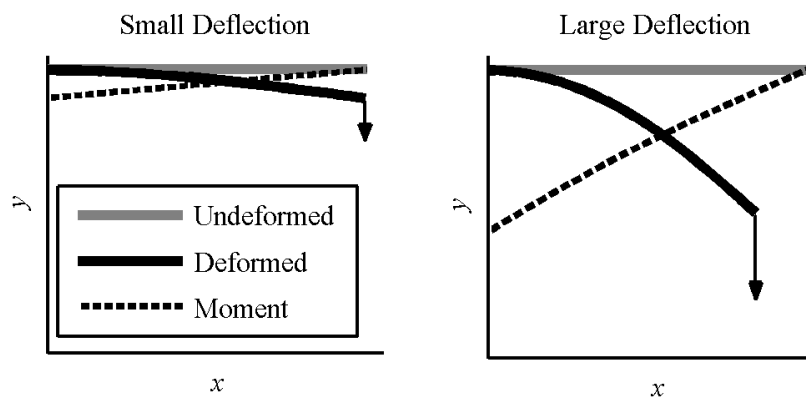

Figure 2. A comparison of the moment profile in small- and largedeflection cantilevered beam bending.

\section{The SMOOTh CuRvature Model}

\section{A. Motivation}

In 1694, Jacob Bernoulli proposed (and solved) the problem of finding the shape of a pre-bent cantilevered beam of length $L$ that would bend into a straight line when loaded with an arbitrarily large force $P$ at the tip [22]. Today the curve is known as the clothoid or Euler spiral. The curvature $\kappa(s)$ of the clothoid curve varies linearly with the arc length $s$ from the base of the cantilever,

$\kappa(s)=\frac{P}{E I}\left(1-\frac{s}{L}\right)$

Here $E$ is the elastic modulus and $I$ is the constant planar moment of the beam area. The clothoid is also a passable approximate solution the more useful problem of finding the deformed shape of an initially straight beam loaded at one end with a large load. This can be seen by examining the nonlinear deformation of a cantilevered beam, as shown in Fig. 2. For a small end load (left), the bending moment will be almost exactly proportional to the distance from the tip of the flexure, as expected. A large end load (right) will produce a non-linear deformation profile, but the moment, plotted as a function of arc length, is still roughly linear. The curvature is directly proportional to bending moment in the beam, $\tau(s)$,

$\kappa(s)=\frac{\tau(s)}{E I}$

For some range of large loads, then, the curvature of a beam can be approximated as some constant plus a linear function of arc length. Horn discussed this approximation in the context of spline curves [23]. The accuracy of this model could be further improved by noting that while the curvature may not be exactly linear, it is certainly smooth, and so might be described with a basis of $n$ Legendre polynomials, $G_{0}(s) \ldots G_{n-l}(s)$. The curvature is expressed as a weighted sum of the bases,

$$
\kappa(s, \underline{q})=q_{0} G_{0}(s)+q_{1} G_{1}(s)+\ldots+q_{n-1} G_{n-1}(s)
$$

The coefficients, $q_{0} \ldots q_{n-1}$, used as a generalized coordinate vector $q$ for describing the deformation of the flexure, are central to the proposed flexure model. Two particular cases will be considered here, corresponding to the models with 2 
and 3 parameters, whose basis functions are Legendre polynomials, translated and scaled to be orthogonal on $[0, L]$,

$\kappa_{2}(s, \underline{q})=q_{0} \frac{1}{L}+q_{1} \frac{2 s-L}{L^{2}}$

$\kappa_{3}(s, \underline{q})=q_{0} \frac{1}{L}+q_{1} \frac{2 s-L}{L^{2}}+q_{2} \frac{6 s^{2}-6 s L+L^{2}}{L^{3}}$

Models of this type, which we will call smooth curvature models, can be used to predict the shape of the flexure in bending, as well as the elastic bending energy. These derivations follow in the next two sections.

\section{B. Flexure Shape}

Joints in a serial robot manipulator are often represented in Denavit-Hartenberg notation, that is, as a parameterized axial, radial and angular transformation from one joint to another. The analogous transformation for a pin joint is a rotation about the joint axis. In the case of a flexure, this transformation corresponds to the translation and rotation from one end of the flexure to the other, as shown in Fig. 3 [12]. This could be written as a matrix, for instance,

$T=\left[\begin{array}{ccc}\cos \left(\varphi_{t i p}\right) & -\sin \left(\varphi_{t i p}\right) & x_{t i p} \\ \sin \left(\varphi_{t i p}\right) & \cos \left(\varphi_{t i p}\right) & y_{t i p} \\ 0 & 0 & 1\end{array}\right]$

The three quantities characterizing this transformation are the flexure tip displacement $\left(x_{t i p}, y_{\text {tip }}\right)$, and the relative angle from the base of the flexure to the tip of the flexure, $\varphi_{\text {tip }}$. They can be written as functions of the polynomial coefficient vector, $q$. The angular profile, $\varphi(s, \underline{q})$, is the integral of the curvature,

$\varphi(s, \underline{q})=\int \kappa(s, \underline{q}) d s$

$\varphi_{2}(s, \underline{q})=q_{0} \frac{s}{L}+q_{1} \frac{s^{2}-s L}{L^{2}}$

$\varphi_{3}(s, \underline{q})=q_{0} \frac{s}{L}+q_{1} \frac{s^{2}-s L}{L^{2}}+q_{2} \frac{2 s^{3}-3 s^{2} L+s L^{2}}{L^{3}}$

At the end of the flexure ( $s=L$ ), the tip angle is equivalent to $q_{0}$ irrespective of the model order. This is a happy side effect of using orthogonal polynomials, as all the higher, non-constant terms must integrate to zero:

$\varphi_{\text {tip }}=\varphi(L, \underline{q})=q_{0}$

The tip position of the flexure can be found by integrating the cosine and sine of the angular profile,

$$
\begin{aligned}
& x_{t i p}=\int_{0}^{L} \cos (\varphi(s, \underline{q})) d s \\
& y_{t i p}=\int_{0}^{L} \sin (\varphi(s, \underline{q})) d s
\end{aligned}
$$

These expressions are transcendental. The second order solution can be solved in terms of Fresnel integrals by

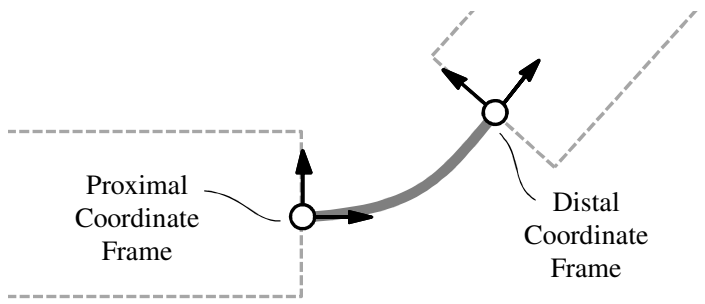

Figure 3. A kinematic model of a flexure consists of a transformation mapping the coordinate frame at the proximal side of the joint to the coordinate frame at the distal side of the joint.

completing the square and using trigonometric addition identities, but it contains discontinuities, and is not practically useful. Further, this strategy does not generalize to the 3 parameter model. Instead, an interpolating approximation was used, so that (11) and (12) can be analytically approximated within some reliable error bounds. In this paper, Chebyshev interpolation [24] was used to approximate the sine and cosine functions as polynomials. There is a trade-off between the domain of interpolation and the computational cost, so maximum flexure rotation was limited to be less than $108^{\circ}$. Alternatively, Gaussian quadrature could be used, essentially interpolating the entire integrand as a polynomial.

\section{Elastic Energy}

Having found the shape of the hinge as a function of $q$, we now turn to finding an expression for the elastic energy in the flexure. The energy stored in an Euler-Bernoulli beam is proportional to the integral of the squared curvature [15]:

$U(\underline{q})=\frac{E I}{2} \int_{0}^{L} \kappa(s, \underline{q})^{2} d s$

If the 2 parameter curvature is used, $U(q)$ evaluates to a weighted sum of the squared parameters.

$$
U_{2}(\underline{q})=\frac{E I}{2} \int_{0}^{L}\left(\frac{q_{0}}{L}+\frac{4 q_{1}(2 s-L)}{L^{2}}\right)^{2} d s=\frac{E I}{2 L}\left(q_{0}^{2}+\frac{q_{1}^{2}}{3}\right)
$$

Because the polynomial basis is orthogonal under convolution over the interval $[0, L]$, there are no cross-terms in this expression. The expression for energy given 3 parameters differs only in the addition of a single term:

$U_{3}(\underline{q})=\frac{E I}{2 L}\left(q_{0}^{2}+\frac{q_{1}^{2}}{3}+\frac{q_{2}^{2}}{5}\right)$

\section{Summary}

The smooth curvature model for flexure hinges has been introduced, based on the observation that the curvature of a flexure can be approximated using a low-dimensional basis of orthogonal polynomials. The position and orientation of the flexure tip can be found relative to its base using only a few model parameters, and these can be used to represent the flexure as a joint in Denavit-Hartenberg form. The elastic energy stored in the bent beam is a weighted sum of the squared flexure parameters. 
The remaining sections will demonstrate that this model can be used to satisfy the two other requirements for a flexure joint model, that is, that the model accurately predicts the equilibrium position of the flexure when an arbitrary force and moment are applied, and that the model accurately predicts variable stiffness effects and buckling due to compressive loads.

\section{DEFLECTION UNDER LOAD}

\section{A. Jacobian Analysis of the Forces on a Robot}

Accurate descriptions of the force exerted on a robot and the resulting deflection are central to many problems in the control and analysis of robot manipulators. The net generalized force $\underline{F}$ on a manipulator experiencing a force $f_{p}$ at some point $\underline{p}$ is given by the Jacobian of that point's coordinates, and the gradient of the potential energy function $U(q)$,

$\underline{F}=\nabla_{q}(\underline{p})^{T} \underline{f}_{p}+\nabla_{q}(U(\underline{q}))$

The generalized force balance equation is only realistic if the generalized coordinates faithfully represent all of the motions that the robot is capable of making. For a flexure hinge, it is important that the motion of flexure tip, as described in the previous section, is accurate, so that the forces and moments transmitted from one link to the next result in a physically realistic deformation of the flexure hinges.

This section considers two tests for benchmarking the ability of the smooth curvature flexure model to predict deformation under load. The first test compares the exact deflection of a cantilevered flexure (using numerically computed elastica curves [25]) to the tip position predicted by the smooth curvature model. The second test computes the deflection of a two-link finger from a tendon-driven elastic gripper developed by the authors. The two flexure hinges in the finger are modeled with finite element flexure models, and with the 3 parameter smooth curvature model, showing that the two models agree despite the vastly reduced parameter space of the smooth curvature model.

\section{B. Tip Deflection of a Loaded Flexure}

A straightforward method of examining the accuracy of a flexible beam is to clamp one end and examine the deflection of the other end when subject to an arbitrary moment $M$ and force $P$ exerted at an angle $\theta$, as shown in Fig. 4. To ensure proper scaling of the results, a non-dimensional form of the beam bending equations should be used, based on these substitutions:

$\tilde{P}=\frac{P L^{2}}{E I}, \tilde{M}=\frac{M L}{E I}, \tilde{\tau}=\frac{\tau L}{E I}, \tilde{s}=\frac{s}{L}, \tilde{x}=\frac{x}{L}, \tilde{y}=\frac{y}{L}$

The dimensionless beam bending equations are equivalent to modeling a beam as having length 1 , and an elastic modulus and cross-sectional moment equal to 1 . The only parameter that is unaffected by this scaling is the beam's angular profile, $\varphi(s)$. Results will be computed for the case when the tip angle, $\varphi_{t i p}$, is equal to $90^{\circ}$, a prototypical test case in the study of large-

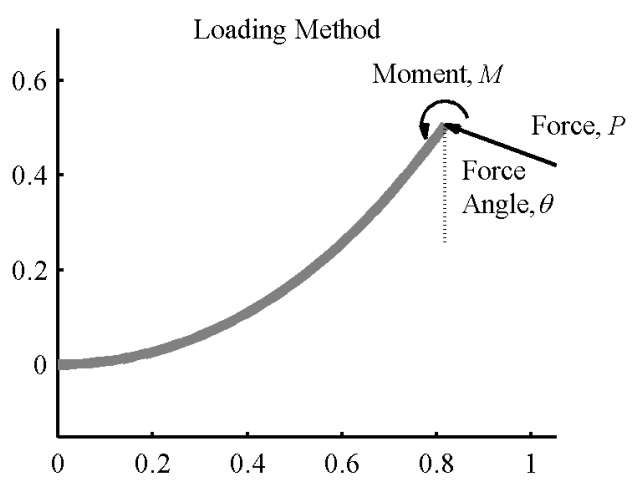

Figure 4. A flexure, loaded at the end by a force and a moment. This figure shows the direction of load, $\theta$.

deformation beam bending ${ }^{1}$. The equilibrium configurations were compared to the exact solution obtained by numerically integrating the large-deformation Euler-Bernoulli equation,

$$
\frac{d}{d \tilde{s}}\left[\begin{array}{c}
\tilde{\tau}(\tilde{s}) \\
\varphi(\tilde{s}) \\
\tilde{x}(\tilde{s}) \\
\tilde{y}(\tilde{s})
\end{array}\right]=\left[\begin{array}{c}
\tilde{P}(\cos (\theta) \cos (\varphi(\tilde{s}))-\sin (\theta) \sin (\varphi(\tilde{s}))) \\
\tilde{\tau}(\tilde{s}) \\
\cos (\varphi(\tilde{s})) \\
\sin (\varphi(\tilde{s}))
\end{array}\right]
$$

This is a restatement of (2), (7), (11) and (12) in differential form, after applying the substitutions from (17). This equation was solved using a Runge-Kutta solver. The tip moment $M$, force angle $\theta$, and tip angle $\varphi_{t i p}$, were specified, and the integral from the tip of the flexure to the based was computed. The tip force, $P$, was found using a bisection search such that the boundary conditions at both ends of the flexure were simultaneously satisfied.

Each force-moment combination $(P, \theta, M)$ that was computed for the exact beam equation was applied to the tip of the smooth curvature flexure model, using the generalized force balance from (16). The Jacobian of the tip coordinates $x_{\text {tip }}, y_{\text {tip }}$ and $\varphi_{\text {tip }}$ was derived from (10), (11) and (12),

$$
\begin{aligned}
& \frac{\partial \varphi_{t i p}}{\partial q_{i}}= \begin{cases}1, & i=0 \\
0, & i \neq 0\end{cases} \\
& \frac{\partial x_{t i p}}{\partial q_{i}}=-\int_{0}^{L} \sin (\varphi(s, \underline{q})) \frac{\partial \varphi_{t i p}}{\partial q_{i}} d s \\
& \frac{\partial y_{t i p}}{\partial q_{i}}=\int_{0}^{L} \cos (\varphi(s, \underline{q})) \frac{\partial \varphi_{t i p}}{\partial q_{i}} d s
\end{aligned}
$$

As in (11) and (12), Chebyshev interpolation was used to produce analytical approximations of (20) and (21). The derivatives of the energy function are much simpler, and can be found from (15),

\footnotetext{
${ }^{1}$ This is a generalization of the rectangular elastica problem posed by Bernoulli, the problem of finding the shape of a cantilevered beam bent at a right angle by a force at the tip [25].
} 


$$
\nabla_{q} U(\underline{q})=\frac{E I}{L}\left[\begin{array}{ccc}
1 & 0 & 0 \\
0 & 1 / 3 & 0 \\
0 & 0 & 1 / 5
\end{array}\right]\left[\begin{array}{l}
q_{0} \\
q_{1} \\
q_{2}
\end{array}\right]
$$

The force balance, computed from (19)-(22), was set to zero to form a system of nonlinear equations, which was solved numerically. Three force-moment combinations were used to compare the two models. These combinations, labeled A, B, and $\mathrm{C}$, are shown in Fig. 5. In case A, a pure bending moment was applied, sufficient to bend the flexure to an angle of $90^{\circ}$. Case $\mathrm{B}$ was a pure force of a magnitude sufficient to bend the flexure to $90^{\circ}$. Case $\mathrm{C}$ was the most complex load, consisting of a moment equal and opposite to the moment applied in combination 1 , counteracted by a force so that the flexure tip angle remained at $90^{\circ}$.

The prediction errors from the smooth curvature models were computed for values of $\theta$ ranging from 20 degrees to 105 degrees (as in Fig. 4). The lower bound of 20 degrees was chosen because the magnitude of the load required to bend a beam to $90^{\circ}$ in case B has a vertical asymptote at $\theta=0$. Thus, the behavior of the flexure becomes increasingly unrealistic in this case. The upper bound of 105 degrees was chosen because the elastica curves generated with the Runge-Kutta solver could not predict buckled configurations, and as the force on the flexure tip becomes increasingly compressive (i.e. $\theta>90^{\circ}$ ), good reference comparisons could not be made. Instead, compressive loads were compared to finite element models in the following subsection.

The positional error of the flexure tip was found, that is, the norm of the vector from the predicted flexure tip to the tip of the numerically computed elastica curve. This is plotted in nondimensional form, meaning that the error is given as a fraction of the flexure length. The angular error is also shown. The errors for the 2 parameter model are shown in Fig. 6, and the errors for the 3 parameter model are shown in Fig. 7. These plots show a number of significant results. First, the errors in case A (pure moment loading) were very small for both the 2 and 3 parameter smooth curvature models. The exact shape one would expect for a beam having a constant bending moment is an arc, a shape that can be exactly reproduced with both models. Thus, the error is correspondingly small. The errors observed in loading cases B and C indicate that the 3 parameter model is significantly more accurate, especially when the flexure is loaded by an opposed force and moment. Most importantly, in every case, the 3 parameter smooth curvature model was within a positional accuracy of 1 percent of the beam length, and an angular accuracy bound of $1^{\circ}$.

\section{Finite Element Comparisons}

One purpose of the smooth curvature model of particular interest to the authors is to enable efficient analysis of manipulators having multiple flexure joints. In previous work, the authors have developed robot hands incorporating polymeric elastic flexure joints [8,9]. These hands are made up of 2-link, tendon-driven fingers, represented in Fig. 8A. In order to evaluate the usefulness of the smooth curvature model for multi-link manipulators, a finite element model was constructed using an object-oriented Matlab library created by

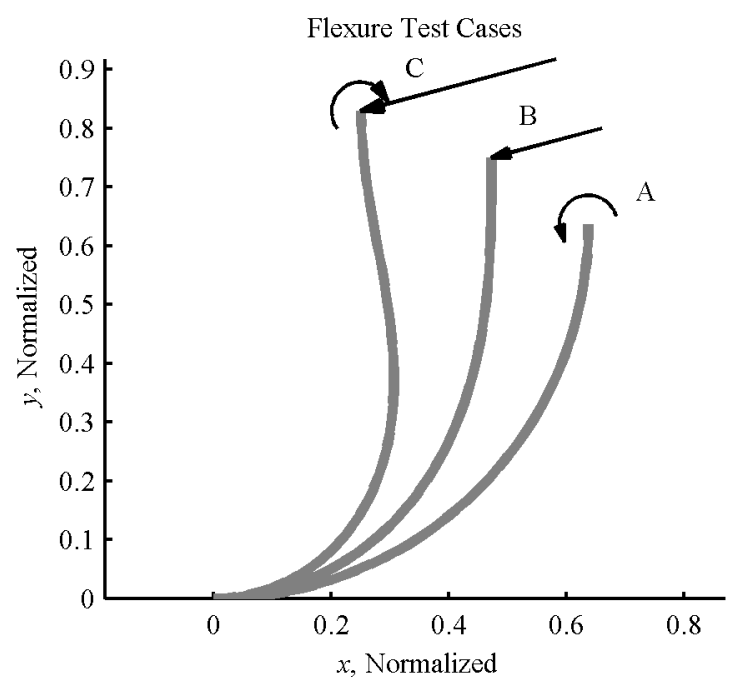

Figure 5. The three loading cases used to test the smooth curvature model.
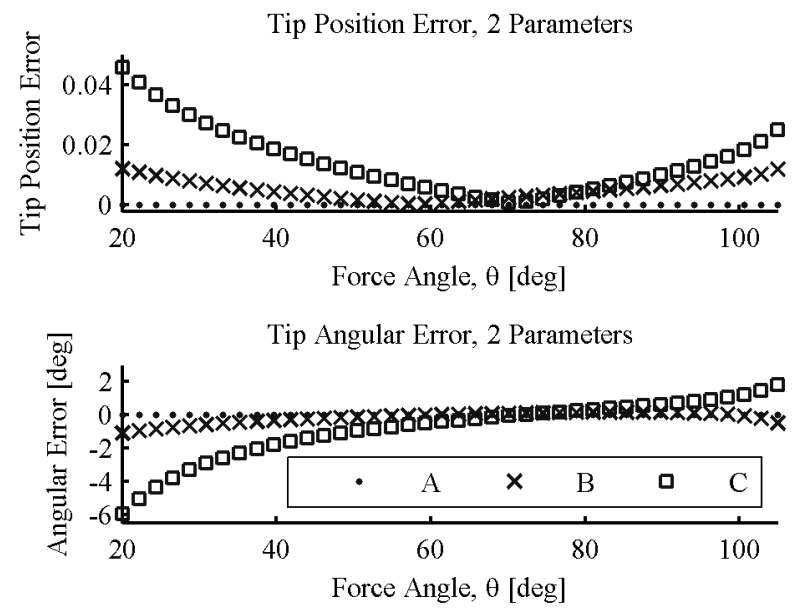

Figure 6. Prediction errors for the 2 parameter smooth curvature model. Cases A, B, and C correspond to the cases in Fig. 5.

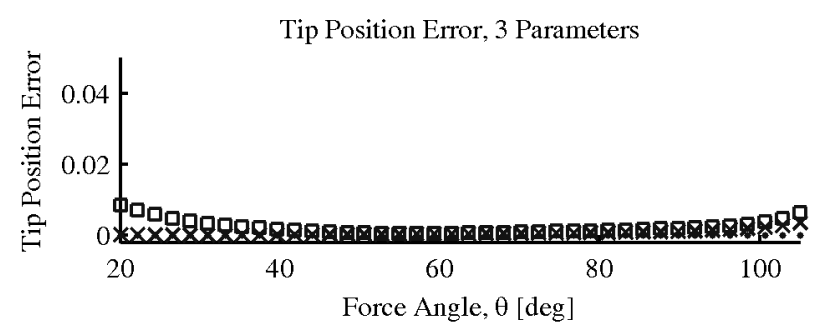

Tip Angular Error, 3 Parameters

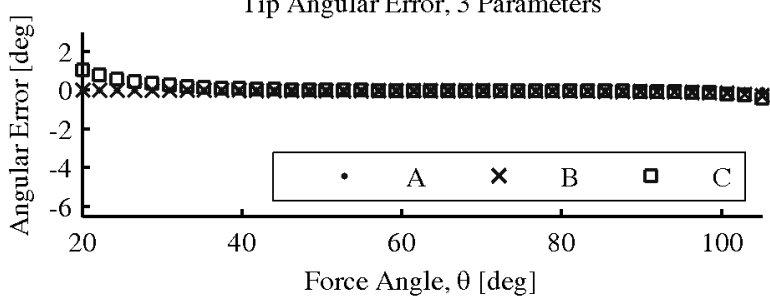

Figure 7. Prediction errors for the 3 parameter smooth curvature model. Cases A, B, and C correspond to the cases in Fig. 5. 


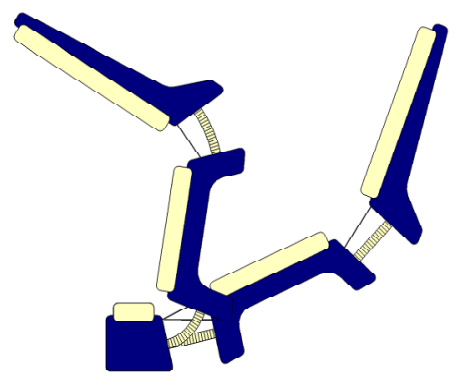

A. Flexure-based robot finger

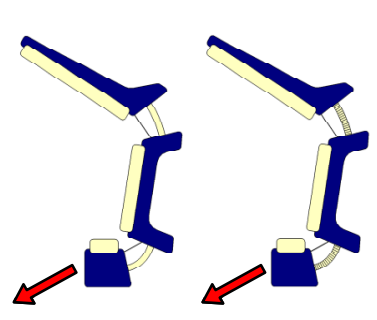

B. Tendon force

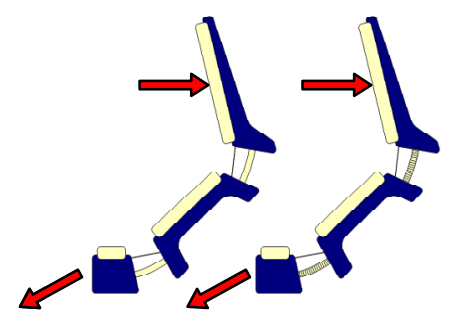

C. Tendon force + pad force

Figure 8. A comparison between FEM and smooth curvature (SC) models: A. The FEM model, no tendon force vs $10 \mathrm{~N}$ tendon force. B. SC model (i) vs FEM (ii), $10 \mathrm{~N}$ tendon force.

C. SC model (iii) vs. FEM (iv), $10 \mathrm{~N}$ force $+0.5 \mathrm{~N}$ force on distal link.

the authors [26]. This model represents each flexure in a fashion similar to the finite element model proposed in [1], consisting of 16 small rotational links. The transformation representing each joint in Denavit-Hartenberg form was the composition of the many resulting rotations and translations making up each finite element.

A model of the finger was also constructed using our 3 parameter smooth curvature flexures. This model uses the joint transformation from (6) and the energy function from (15) to describe the joint behavior in terms of three generalized coordinates per joint, for a total of 6 . The finite element model, by comparison, had a total of 32 generalized coordinates. Both models were subjected to two different loading conditions: in the first condition, the finger was actuated with a single tendon connected to the distal link, as in Fig. 8B. The second condition, depicted in Fig. 8C, included the same tendon force and a horizontal force applied to the center of the pad on the distal link. In both cases, the generalized force balance was computed using (16), computing the kinematics of the tendon and the center of the distal pad using a composition of geometric joint and link transformations.

The results of the test are shown in Fig. 8 and Table I, which describes the agreement between the smooth curvature and finite element models as to the position and orientation of the center of the distal link. The results show that the displacement of the distal link pad as calculated by the smooth curvature model is within $0.1 \%$ of the position predicted by the finite element model. The angular agreement is similarly within a tenth of a degree in both cases. Attempts at visual comparison between the FEM and smooth curvature models by overlaying the two were unsuccessful, because they were almost indistinguishable to the eye.

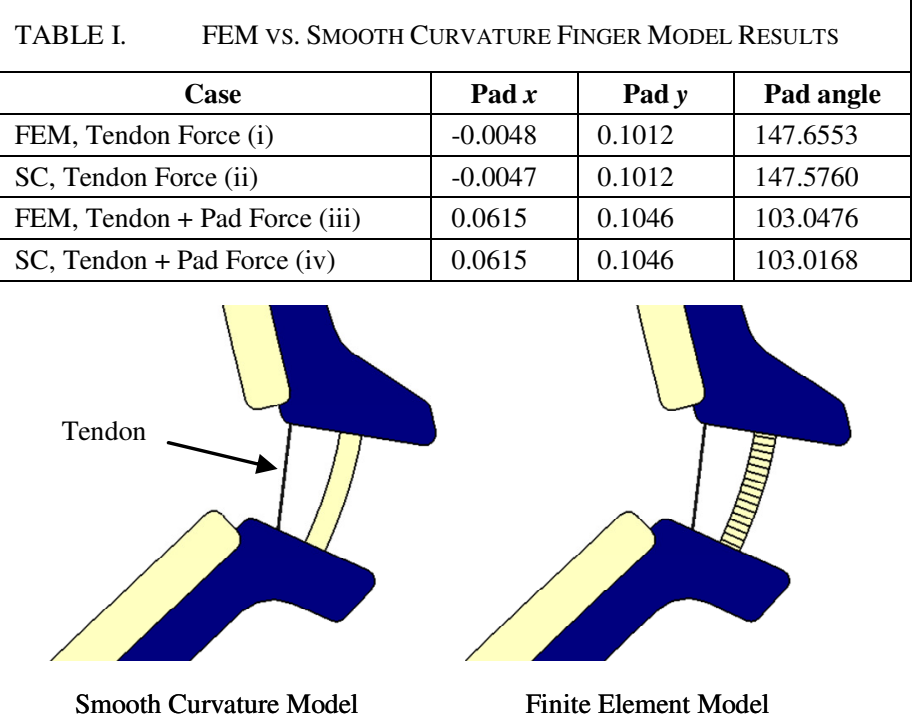

Figure 9. A single tendon-driven joint, modeled using the smooth curvature model (left) and finite rotational elements (right).

\section{Summary}

For both a single flexure hinge and a two-joint manipulator, the smooth curvature model has been shown to accurately predict the deformation of a manipulator under a wide range of forces and moments. This is interesting and new because most methods of accurately solving large-deflection beam bending problems involve numerical integration, or the breaking down of a beam into many finite elements. Because the smooth curvature model achieves a useful degree of accuracy with only three parameters per joint, calculation of dynamics and statics for control or motion planning is a much simpler process.

\section{STIFFNESS AND BUCKLING}

\section{A. Stiffness of a Loaded Elastic Structure}

One major difference between the well-studied problem of flexible-link manipulators and the newer field of flexurejointed manipulators is the relatively increased importance of buckling in flexure joints. The flexure joints shown in Fig. 9 show a typical tendon/flexure actuation scheme. The tendon exerts a force in tension, which is balanced by a compressive force in the flexure, unless a parallel load path exists. This compressive force is quite large, and can easily approach the Euler buckling load of a thin flexure (the load at which the lateral stiffness of the flexure is zero). Unlike structural columns, buckling does not represent a necessarily undesirable effect. The fact that the flexure is buckled just means that its rotational stiffness is very low. Most pin joints, for example, have zero rotational stiffness and this is not an impediment to their use in robots. However, this change in stiffness as a function of load is critically important in some robotic tasks. For example, a change in joint stiffness will affect computed torque control models. Additionally, some tasks such as grasping and manipulation rely on the elastic stability of the 
whole system; a buckling mode could be harmless, or it could correspond to configuration in which a grasped object twists out of its gripped position [20]. As a result of all these concerns, it is important that a flexure model provide a reasonable model of elastic buckling.

This section briefly describes the ability of the smooth curvature model to predict elastic buckling in a flexure using the Hessian of the flexure kinematics. As a proxy case for comparing the continuum behavior of a flexure to the smooth curvature model, the smooth curvature model will be used to predict buckling in compression by finding the smallest compressive load for which the generalized stiffness matrix is singular. This result can be compared to Euler's buckling load formula. The 2 and 3 parameter models produce successively better approximations of buckling.

\section{B. Continuum vs. Discrete Buckling}

A continuum structure is said to buckle when it has zero (or negative) stiffness in some direction, so that a small perturbation to the structure's shape is met by a destabilizing force, rather than a restoring force. The compressive load $P_{\text {crit }}$ at which a clamped-free beam should buckle is given by Euler's well-known formula [27]:

$$
\frac{P_{\text {crit }} L^{2}}{E I}=\left(\frac{\pi}{2}\right)^{2}=2.4674 \ldots
$$

The concept analogous to elastic buckling in a generalized coordinate model has to do with the generalized stiffness matrix obtained by taking the gradient of the generalized force balance from (16) with respect to $q$,

$$
\mathbf{K}=\nabla_{q} \nabla_{q}(\underline{p})^{T} \underline{f}_{p}+\nabla_{q} \nabla_{q}(U(\underline{q}))
$$

Stiffness is a function of the Hessian of the coordinates where force is applied, and of the Hessian of the energy, $U(q)$. When this stiffness matrix has an eigenvalue that is zero or negative, it buckles. In other words, there will exist some eigenvector $\delta q$, which, when applied to the robot as a perturbation, will produce a destabilizing force. This could also be thought of as a test for the convexity of the total energy in the robot.

We will derive the generalized stiffness matrix for the smooth curvature model, when the flexure is loaded in the $-x$ direction with a force, $P$. Thus, the contact point $\underline{p}$ from (24) above is the scalar $x_{\text {tip }}$, as described in (8). The Hessian elements can be calculated from the Jacobian of $x_{t i p}$ in (20),

$$
\frac{\partial^{2} x_{t i p}}{\partial q_{i} \partial q_{j}}=-\int_{0}^{L}\left[\cos (\phi(s)) \frac{\partial \phi}{\partial q_{i}} \frac{\partial \phi}{\partial q_{j}}+\sin (\phi(s)) \frac{\partial^{2} \phi}{\partial q_{i} \partial q_{j}}\right] d s
$$

When the flexure is straight, $q_{0}=q_{1}=q_{2}=0$. In this configuration, (25) can be simplified, because the sine term disappears and the cosine term approaches one,

$$
\frac{\partial^{2} x_{t i p}}{\partial q_{i} \partial q_{j}}=-\int_{0}^{L} \frac{\partial \phi}{\partial q_{i}} \frac{\partial \phi}{\partial q_{j}} d s
$$

These are polynomials, which can be evaluated to compute the Hessian of $x_{\text {tip }}$ with respect to the generalized coordinates. For the 2 parameter model, the Hessian is a 2 by 2 matrix,

$$
\nabla_{q} \nabla_{q}\left(x_{\text {tip }}\right)=L\left[\begin{array}{cc}
1 / 3 & -1 / 12 \\
-1 / 12 & 1 / 30
\end{array}\right]
$$

The stiffness due to potential energy can be found by taking the Hessian of the energy function derived in (14),

$$
\nabla_{q} \nabla_{q}(U(\underline{q}))=\frac{E I}{L}\left[\begin{array}{cc}
1 & 0 \\
0 & 1 / 3
\end{array}\right]
$$

The coordinate Hessian and the energy Hessian can be substituted back into (24),

$$
\mathbf{K}=\frac{E I}{L}\left[\begin{array}{cc}
1 & 0 \\
0 & 1 / 3
\end{array}\right]-P L\left[\begin{array}{cc}
1 / 3 & -1 / 12 \\
-1 / 12 & 1 / 30
\end{array}\right]
$$

The buckling load of the 2 parameter model, $P_{2}$, is the value of $P$ for which the determinant of $\mathbf{K}$ is zero, indicating that the matrix has an eigenvalue equal to zero,

$$
\left|\begin{array}{cc}
\frac{E I}{P_{2} L^{2}}-\frac{1}{3} & \frac{1}{12} \\
\frac{1}{12} & \frac{E I}{3 P_{2} L^{2}}-\frac{1}{30}
\end{array}\right|=0
$$

The smallest root of this polynomial is the most physically meaningful, as it represents the load at which the unconstrained flexure will buckle,

$$
\frac{P_{2} L^{2}}{E I}=2.4860 \ldots
$$

This is only $0.75 \%$ larger than the true value reported by the continuum model in (23). This exercise can be repeated for the three parameter model, to find the predicted buckling load, $P_{3}$,

$$
\left|\begin{array}{ccc}
\frac{E I}{P_{3} L^{2}}-\frac{1}{3} & \frac{1}{12} & \frac{1}{60} \\
\frac{1}{12} & \frac{E I}{3 P_{3} L^{2}}-\frac{1}{30} & 0 \\
\frac{1}{60} & 0 & \frac{E I}{5 P_{3} L^{2}}-\frac{1}{210}
\end{array}\right|=0
$$

The resulting buckling load prediction is within $0.02 \%$ of the value predicted by Euler's beam buckling formula,

$$
\frac{P_{3} L^{2}}{E I}=2.4677 \ldots
$$

\section{Discussion}

The results of this brief study indicate that the smooth curvature model has no difficulty predicting the stiffness of a straightened flexure hinge as a function of load. A more general argument, too long to be presented here, would also cover the stiffness of a constrained flexure, such as one which is pinned or clamped at the distal end. Although the effect of constraints on the generalized stiffness is lengthier to describe, 
the 3 parameter model can successfully describe these cases. Finally, it is worth mentioning that although stiffness prediction of a flexure undergoing large deformation has not been presented here, the smooth curvature model will predict the stiffness matrix for large deformations, as we have shown in [21].

\section{CONCLUSIONS}

\section{A. In Summary}

In this paper, we have presented a model for flexible links that is accurate for large deformations, so that it can be used for the special case of flexible links as flexure hinges. These models are compatible with all of the standard tools used for manipulator analysis, because they are in a form where the shape of the joint and the elastic energy of the joint can be entirely described by a set of generalized coordinates. We have demonstrated that a flexure can be described to a high level of accuracy using only three parameters - arguably the minimum number of parameters capable of describing a flexure with three independent end conditions, $x_{\text {tip }}, y_{\text {tip }}$ and $\varphi_{\text {tip }}$. This model is useful for "zeroth" order descriptions (shape and energy), first order descriptions (local deformation and force), and for second order descriptions (buckling configurations and stiffness) of mechanical behavior of flexible members undergoing large deformations under loads.

\section{B. Future Directions}

The smooth curvature flexure model presents several obvious directions for further study. First, this model does not take into account axial deformation, which is important in accurately analyzing parallel mechanisms. We are confident that this method can be extended to describe such mechanisms through the addition of one or two axial deformation modes.

Second, it is worth noting that although models of planar beam bending are quite useful, many flexures admit a great deal of out-of-plane motion. This behavior can be characterized by modal models similar to the one presented here. We are working on extensions of smooth curvature models to three dimensions, including possible techniques for coping with the greatly increased complexity of describing non-commutative spatial rotations.

\section{REFERENCES}

[1] E. Bayo, "A finite-element approach to control the end-point motion of a single-link flexible robot," Journal of Robotic Systems, vol. 4(1), pp. 6375, 1986.

[2] H. Asada, Z.-D. Ma, and H. Tokumaru, "Inverse Dynamics of Flexible Robot Arms: Modeling and Computation for Trajectory Control", J. Dynamic Systems, Measurement, and Control vol.112(2), pp. 177-186, 1990.

[3] Zheng-Hua Luo, "Direct strain feedback control of flexible robot arms: new theoretical and experimental results," IEEE Transactions on Automatic Control, vol.38(11), pp.1610-1622, 1993.

[4] A. Hemami, "Studies on a light weight and flexible robot manipulator," Robotics, vol. 1(1), pp. 27-36, 1985.
[5] S. Sur and R.M. Murray, "An Experimental Comparison of Tradeoffs in Using Compliant Manipulators for Robotic Grasping Tasks,"Proceedings of the 1997 IEEE International Conference on Robotics and Automation, pp. 1807-1814, 1997.

[6] F. Lotti and G. Vassura, "A Novel Approach to Mechanical Design of Articulated Fingers for Robotic Hands," Proceedings of the 2002 IEEE International Conference on Intelligent Robots and Systems, pp. 16871692, 2002.

[7] F. Lotti, P. Tiezzi, G. Vassura, L. Biagiotti, G. Palli, C. Melchiorri, "Development of UB Hand 3: Early Results," Proceedings of the 2005 IEEE International Conference on Robotics and Automation, pp. $4499-$ 4504, 2005.

[8] A. Dollar and R. Howe, "The Highly Adaptive SDM Hand: Design and Performance Evaluation," Int. Journal of Robotics Research, v.29, pp. 585-597, 2010.

[9] A. Dollar and R. Howe, "Towards grasping in unstructured environments: Grasper compliance and configuration optimization," Advanced Robotics, v. 19, n. 5, pp. 523-543, 2005.

[10] R. Digiantonio and G. Lawrence, "Two-shot molding of thermoplastic elastomers," Proceedings of the 1992 SPE Annual Technology Conference, pp. 851-853, 1992

[11] R. Merz, F. Prinz, K. Ramaswami, M. Terk, L. Weiss, "Shape deposition manufacturing," Proceedings of the Solid Freeform Fabrication Symposium, v. 5, pp.1-8, 1994

[12] M. Hannan and I. Walker, "Kinematics and the implementation of an elephant's trunk manipulator and other continuum style robots," Journal of Robotics Systems, v. 20, n. 2, pp. 45-63, 2003

[13] M. Ciocarlie and P. Allen, "A Design and Analysis Tool for Underactuated Compliant Hands," Proceedings of the 2009 IEEE/RSJ Int. Conf. on Intelligent Robotics and Systems, pp. 5235-5239, 2009

[14] A. Midha, T. Norton, and L. Howell, "On the nomenclature, classification, and abstractions of compliant mechanisms," Trans. of the ASME, v. 116, n. 1, pp.270-280, 1994

[15] S. Timoshenko and D. Young, Advanced Dynamics, p. 204, McGrawHill, 1948.

[16] A. DeLuca and B. Siciliano, "Closed-form dynamic model of planar multilink lightweight robots," IEEE Trans. on Systems, Man and Cybernetics, v. 21, n. 4, pp. 826-839, 1991

[17] G. Chirikjian and J. Burdick, "A modal approach to hyper-redundant manipulator kinematics," IEEE Trans. on Robotics and Automation, v. 10, n. 3, pp. 343-354, 1994

[18] N. Lobontiu, "Distributed-Parameter dynamic model and optimized design of a four-link pendulum with flexure hinges," Mechanism and Machine Theory, v. 36, pp. 653-669, 2001

[19] N. Lobontiu and E. Garcia, "Two microcantilever designs: lumpedparameter model for static and modal analysis," Journal of Microelectromechanical Systems, v. 13, 2004

[20] H. Hanafusa and H. Asada, "Stable Prehension by a Robot Hand with Elastic Fingers," Proceedings of the 7th International Symposium on Industrial Robots, pp. 361-368, 1977.

[21] L. Odhner and A. Dollar, "Fast, accurate models for predicting the compliance of elastic flexure-jointed robots," ASME Internat. Design Engineering Technical Conf and Computers and Information in Engineering Conf., 2010.

[22] R. Levien, "The Euler spiral: a mathematical history," Technical Report No. UCB/EECS-2008-111, University of California at Berkeley, 2008

[23] B. Horn, "The Curve of Least Energy," ACM Trans. On Mathematical Software, vol. 9, n. 4, pp. 441-460, 1983

[24] W. Press, S. Teukolsky, W. Vetterling, and B. Flannery, Numerical Recipes in C, $2^{\text {nd }}$ ed., p. 190, Cambridge University Press, 1992.

[25] R. Levien, "The elastica: a mathematical history," Technical Report No. UCB/EECS-2008-103, University of California at Berkeley, 2008

[26] L. Odhner and A. Dollar, "The Freeform Manipulator Analysis Tool" [Online]. Avaliable: http://www.eng.yale.edu/grablab/fmat/

[27] S. Crandall, C. Dahl and T. Lardner, An Introduction to the Mechanics of Solids, $2^{\text {nd }} \quad$ ed., p. 587, McGraw-Hill, 1999. 